\title{
Multiple solutions for fractional $p$-Laplace equation with concave-convex nonlinearities
}

\section{Qiang Chen ${ }^{1 *}$, Caisheng Chen ${ }^{2}$ and Yanling Shi'}

${ }^{*}$ Correspondence: chq623@sohu.com

'School of Mathematics and Physics, Yancheng Institute of Technology, Yancheng, P.R. China Full list of author information is available at the end of the article

\section{Abstract}

In this paper, we investigate the existence of solutions for the fractional $p$-Laplace equation

$$
(-\Delta)_{p}^{s} u+v(x)|u|^{p-2} u=h_{1}(x)|u|^{q-2} u+h_{2}(x)|u|^{r-2} u \quad \text { in } \mathbb{R}^{N},
$$

where $N>s p, 0<s<1<p, 1<q<p<r<p_{s}^{*}:=\frac{N p}{N-s p}$, and the potential function $V(x)>0$ and $h_{1}(x), h_{2}(x)$ are allowed to change sign in $\mathbb{R}^{N}$. By using variant fountain theorem, we prove that the above equation admits infinitely many small and high energy solutions.

MSC: 35J20; 35J70; 35J91

Keywords: Fractional $p$-Laplacian equations; Variational methods; Variant fountain theorem

\section{Introduction and main result}

In this paper, we consider the existence and multiplicity of solutions for the following elliptic problem:

$$
(-\Delta)_{p}^{s} u+V(x)|u|^{p-2} u=h_{1}(x)|u|^{q-2} u+h_{2}(x)|u|^{r-2} u \quad \text { in } \mathbb{R}^{N}
$$

where $(-\Delta)_{p}^{s}$ is the fractional $p$-Laplacian operator with $0<s<1<p$ and $s p<N, 1<q<$ $p<r<p_{s}^{*}:=\frac{N p}{N-s p}$ and potential function $V(x)>0, h_{1}$ and $h_{2}$ are sign-changing weight functions. The exact assumptions will be given below.

The fractional $p$-Laplacian operator $(-\Delta)_{p}^{s}$ is defined along a function $u \in C_{0}^{\infty}\left(\mathbb{R}^{N}\right)$ as follows:

$$
(-\Delta)_{p}^{s} u(x)=2 \lim _{\varepsilon \rightarrow 0^{+}} \int_{\mathbb{R}^{N} \backslash B_{\varepsilon}(x)} \frac{|u(x)-u(y)|^{p-2}(u(x)-u(y))}{|x-y|^{N+p s}} d y
$$

for $x \in \mathbb{R}^{N}$, where $B_{\varepsilon}(x)=\left\{y \in \mathbb{R}^{N}:|x-y|<\varepsilon\right\}$, see [1-3] and the references therein.

In the last years, since the nonlinear equations involving fractional powers of the Laplacian played an increasingly important role in physics, probability, and finance, a great at-

(c) The Author(s) 2020. This article is licensed under a Creative Commons Attribution 4.0 International License, which permits use, sharing, adaptation, distribution and reproduction in any medium or format, as long as you give appropriate credit to the original author(s) and the source, provide a link to the Creative Commons licence, and indicate if changes were made. The images or other third party material in this article are included in the article's Creative Commons licence, unless indicated otherwise in a credit line to the material. If material is not included in the article's Creative Commons licence and your intended use is not permitted by statutory regulation or exceeds the permitted use, you will need to obtain permission directly from the copyright holder. To view a copy of this licence, visit http://creativecommons.org/licenses/by/4.0/. 
tention has been focused on the study of problems involving the fractional Laplacian. So there has been a lot of interest in the study of the fractional Schrödinger equation

$$
(-\Delta)^{s} u+V(x) u=f(x, u), \quad x \in \mathbb{R}^{N},
$$

where the nonlinearity $f$ satisfies some general conditions, see for instance [4-19] and the references therein.

More recently, Xiang et al. [3] investigated the fractional $p$-Laplacian equation

$$
(-\Delta)_{p}^{s} u+V(x)|u|^{p-2} u=\lambda a(x)|u|^{r-2} u-b(x)|u|^{q-2} u \quad \text { in } \mathbb{R}^{N},
$$

where $\lambda>0, p<r<\min \left\{q, p_{s} *\right\}$, and $a(x)$ and $b(x)$ are related by the condition $a(a / b)^{(r-p)(q-r)} \in L^{N / p s}\left(\mathbb{R}^{N}\right)$. By using a direct variational method and the mountain pass theorem, the authors proved the existence of two nontrivial weak solutions of (1.4) for $\lambda>\lambda^{*}\left(\lambda^{*}>0\right.$ is a given constant).

There are also a lot of works about problem (1.3) with concave-convex nonlinearities. Goyal and Sreenadh in [20] considered the following $p$-fractional Laplace equation:

$$
\left\{\begin{array}{l}
(-\Delta)_{p}^{s} u=\lambda h(x)|u|^{q-2} u+b(x)|u|^{r-2} u \quad \text { in } \Omega \\
u=0 \text { on } \partial \Omega
\end{array}\right.
$$

where $\Omega$ is a bounded domain in $\mathbb{R}^{N}$ with Lipschitz boundary, $p \geq 2, n>p \alpha, 1<q<p<$ $r<p_{s}^{*}, \lambda>0, h$ and $b$ are sign-changing smooth functions. They proved that there exists $\lambda_{0}>0$ such that problem (1.5) has at least two nonnegative solutions for all $\lambda \in\left(0, \lambda_{0}\right)$.

In [21], the authors considered the problem as follows:

$$
M\left([u]_{s, p}^{p}\right)(-\Delta)_{p}^{s} u=\lambda h_{1}(x)|u|^{q-2} u+h_{2}(x)|u|^{r-2} u+h(x) \quad \text { in } \mathbb{R}^{N},
$$

where $M(t)=a+b t^{\theta-1}, \theta>1, a, b \geq 0, a+b>0, \lambda>0$, and $1<q<p<\theta p<r<p_{s}^{*}$. The functions $h_{1}(x), h_{2}(x)$, and $h(x)$ may change sign on $\mathbb{R}^{N}$. Note that problem (1.6) is reduced to the fractional $p$-Laplacian equation with $a=1$ and $b=0$. Under some suitable conditions, they obtained the existence of two nontrivial entire solutions by applying the mountain pass theorem and Ekeland's variational principle.

In this paper, we are interested in the multiplicity of solutions to equation (1.1) and find sufficient conditions to guarantee the existence of infinitely many solutions.

The present article is motivated by the papers [6,7], as well as by the fact that we do not find in the literature any paper dealing with the existence of infinitely many solutions to equation (1.1). The main tools employed in our works are the variant fountain theorems established in [22]. They are effective tools for studying the existence of infinitely many large or small energy solutions. Moreover, the results about the existence of solutions in the above papers are all related to the number $\lambda$. So we are also interested in whether the restriction on $\lambda$ can be taken out.

Throughout this paper, we make the following assumptions:

$\left(H_{1}\right) \quad V(x) \in C\left(\mathbb{R}^{N}\right)$ and there exists $V_{0}>0$ such that $V(x) \geq V_{0}$ in $\mathbb{R}^{N}$;

$\left(H_{2}\right) h_{1}(x) \in L^{\tau}\left(\mathbb{R}^{N}\right)$, where $\tau \in\left[\frac{p_{s}^{*}}{p_{s}^{*}-q}, \frac{p}{p-q}\right]$ is a positive constant;

$\left(H_{3}\right)$ There exists $\mu \in\left[\frac{p_{s}^{*}}{p_{s}^{*}-r}, \infty\right)$ such that $h_{2}(x) \in L^{\mu}\left(\mathbb{R}^{N}\right)$; 
$\left(H_{3}^{\prime}\right) h_{2}(x) \in L^{\infty}\left(\mathbb{R}^{N}\right)$ and $h_{2}(x) \rightarrow 0$ as $|x| \rightarrow \infty$.

Our main results in this paper are stated as follows.

Theorem 1.1 Assume $\left(H_{1}\right),\left(H_{2}\right),\left(H_{3}\right)\left(\right.$ or $\left.\left(H_{3}^{\prime}\right)\right)$, and $h_{1}(x)>0$, then problem (1.1) admits infinitely many small energy solutions $u_{k} \in E$ satisfying $I\left(u_{k}\right) \rightarrow 0^{-}$as $k \rightarrow \infty$.

Theorem 1.2 Let $\left(H_{1}\right),\left(H_{2}\right)$, and $\left(H_{3}\right)\left(\right.$ or $\left.\left(H_{3}^{\prime}\right)\right)$ hold. If $h_{2}(x)>0$ is satisfied, then problem (1.1) possesses infinitely many high energy solutions $u_{k} \in E$ such that $I\left(u_{k}\right) \rightarrow \infty$ as $k \rightarrow \infty$.

The functional $I$ that appeared in Theorems 1.1-1.2 is the energy functional for problem (1.1), which will be given below.

By combining Theorems 1.1-1.2, the following corollary is immediate.

Corollary 1.3 Let $\left(H_{1}\right),\left(H_{2}\right)$, and $\left(H_{3}\right)\left(\right.$ or $\left.\left(H_{3}^{\prime}\right)\right)$ hold. If $h_{1}(x), h_{2}(x)>0$, then problem $(1.1)$ has two sequences $\left\{u_{k}\right\}$ and $\left\{\bar{u}_{k}\right\}$ of nontrivial solutions such that

$$
I\left(u_{k}\right) \rightarrow 0^{-}, \quad I\left(\bar{u}_{k}\right) \rightarrow \infty \quad \text { as } k \rightarrow \infty .
$$

The rest of the paper is organized as follows. In the forthcoming section, we set up the variational framework for (1.1) and state the variant fountain theorems that will be used later. In Sect. 3, we study problem (1.1) and give the proof of Theorem 1.1. Section 4 is devoted to the proof of Theorem 1.2.

\section{Preliminaries}

First of all, we give some basic results of fractional Sobolev space that will be used in the next sections. Let $0<s<1<p$ be real numbers. The fractional Sobolev space $W^{s, p}\left(\mathbb{R}^{N}\right)$ is defined as follows:

$$
W^{s, p}\left(\mathbb{R}^{N}\right)=\left\{u \in L^{p}\left(\mathbb{R}^{N}\right): \int_{\mathbb{R}^{N} \times \mathbb{R}^{N}} \frac{|u(x)-u(y)|^{p}}{|x-y|^{N+s p}} d x d y<\infty\right\}
$$

equipped with the norm

$$
\|u\|_{W^{s, p}\left(\mathbb{R}^{N)}\right.}=\left(\|u\|_{L^{p}\left(\mathbb{R}^{N}\right)}^{p}+\int_{\mathbb{R}^{N} \times \mathbb{R}^{N}} \frac{|u(x)-u(y)|^{p}}{|x-y|^{N+s p}} d x d y\right)^{\frac{1}{p}} .
$$

Then $\left(W^{s, p}\left(\mathbb{R}^{N}\right),\|u\|_{W^{s, p}\left(\mathbb{R}^{N}\right)}\right)$ is a uniformly convex Banach space and the embedding

$$
W^{s, p}\left(\mathbb{R}^{N}\right) \hookrightarrow L^{t}\left(\mathbb{R}^{N}\right)
$$

is continuous for $t \in\left[p, p_{s}^{*}\right]$. Moreover, the embedding is locally compact whenever $1 \leq$ $t<p_{s}^{*}$, see [1] for details.

For our problem (1.1), consider the subspace $X \subset W^{s, p}\left(\mathbb{R}^{N}\right)$ given by

$$
X=\left\{u \in W^{s, p}\left(\mathbb{R}^{N}\right): \int_{\mathbb{R}^{N}} V(x)|u|^{p} d x<\infty\right\}
$$


Then $X$ is a separable Banach space with the norm

$$
\|u\|=\left(\int_{\mathbb{R}^{N} \times \mathbb{R}^{N}} \frac{|u(x)-u(y)|^{p}}{|x-y|^{N+s p}} d x d y+\int_{\mathbb{R}^{N}} V(x)|u|^{p} d x\right)^{\frac{1}{p}} .
$$

As usual, for $t \geq 1$, we let

$$
|u|_{t}=\left(\int_{\mathbb{R}^{N}}|u|^{t} d x\right)^{\frac{1}{t}}, \quad u \in L^{t}\left(\mathbb{R}^{N}\right)
$$

By the embedding $X \hookrightarrow L^{t}\left(\mathbb{R}^{N}\right)$, we know that there exists a constant $S_{t}>0$ such that

$$
|u|_{t} \leq S_{t}\|u\|, \quad t \in\left[p, p_{s}^{*}\right]
$$

Definition 2.1 A function $u \in X$ is said to be a (weak) solution of (1.1) if, for any $v \in X$, we have

$$
\begin{gathered}
\int_{\mathbb{R}^{N} \times \mathbb{R}^{N}} \frac{|u(x)-u(y)|^{p-2}(u(x)-u(y))(v(x)-v(y))}{|x-y|^{N+s p}} d x d y+\int_{\mathbb{R}^{N}} V(x)|u|^{p-2} u v d x \\
=\int_{\mathbb{R}^{N}} h_{1}(x)|u|^{q-2} u v d x+\int_{\mathbb{R}^{N}} h_{2}(x)|u|^{r-2} u v d x .
\end{gathered}
$$

Let $I(u): X \rightarrow \mathbb{R}$ be the energy functional associated with (1.1) defined by

$$
I(u)=\frac{1}{p}\|u\|^{p}-\frac{1}{q} \int_{\mathbb{R}^{N}} h_{1}(x)|u|^{q} d x-\frac{1}{r} \int_{\mathbb{R}^{N}} h_{2}(x)|u|^{r} d x .
$$

Using (2.6), it follows from conditions $\left(H_{1}\right),\left(H_{2}\right)$, and $\left(H_{3}\right)$ (or $\left.\left(H_{3}^{\prime}\right)\right)$ that the functional $I$ is well defined and $I \in C^{1}(X, \mathbb{R})$ with

$$
\begin{aligned}
I^{\prime}(u) v= & \int_{\mathbb{R}^{N} \times \mathbb{R}^{N}} \frac{|u(x)-u(y)|^{p-2}(u(x)-u(y))(v(x)-v(y))}{|x-y|^{N+s p}} d x d y \\
& +\int_{\mathbb{R}^{N}} V(x)|u|^{p-2} u v d x-\int_{\mathbb{R}^{N}} h_{1}(x)|u|^{q-2} u v d x-\int_{\mathbb{R}^{N}} h_{2}(x)|u|^{r-2} u v d x
\end{aligned}
$$

for any $v \in X$. It is standard to verify that the weak solutions of (1.1) correspond to the critical points of $I$.

Finally, we give the variant fountain theorems.

Let $X$ be a Banach space with the norm $\|\cdot\|$ and $X=\overline{\bigoplus_{j=1}^{\infty} X_{j}}$ with $\operatorname{dim} X_{j}<\infty, j \in \mathbb{Z}$. Define

$$
Y_{k}=\bigoplus_{j=1}^{k} X_{j}, \quad Z_{k}=\overline{\bigoplus_{j=k}^{\infty} X_{j}}
$$

Consider the following $C^{1}$-functional $I_{\lambda}: X \rightarrow \mathbb{R}$ defined by

$$
I_{\lambda}(u):=A(u)-\lambda B(u), \quad \lambda \in[1,2] .
$$

The following two variant fountain theorems were established in [22]. 
Theorem 2.2 Assume that the functional $I_{\lambda}$ defined above satisfies

$\left(A_{1}\right) I_{\lambda}$ maps bounded sets into bounded sets uniformly for $\lambda \in[1,2]$, and $I_{\lambda}(-u)=I_{\lambda}(u)$ for all $(\lambda, u) \in[1,2] \times X$;

$\left(A_{2}\right) B(u) \geq 0$ for all $u \in X$, and $B(u) \rightarrow \infty$ as $\|u\| \rightarrow \infty$ on any finite dimensional subspace of $X$;

$\left(A_{3}\right)$ There exist $\rho_{k}>r_{k}>0$ such that

$$
a_{k}(\lambda):=\inf _{u \in Z_{k},\|u\|=\rho_{k}} I_{\lambda}(u) \geq 0, \quad b_{k}(\lambda):=\max _{u \in Y_{k},\|u\|=r_{k}} I_{\lambda}(u)<0, \quad \forall \lambda \in[1,2]
$$

and

$$
d_{k}(\lambda):=\inf _{u \in Z_{k},\|u\| \leq \rho_{k}} I_{\lambda}(u) \rightarrow 0, \quad \text { as } k \rightarrow \infty \text { uniformly for } \lambda \in[1,2] .
$$

Then there exist $\lambda_{n} \rightarrow 1, u_{n}\left(\lambda_{n}\right) \in Y_{n}$ such that

$$
\left.I_{\lambda_{n}}^{\prime}\right|_{Y_{n}}\left(u\left(\lambda_{n}\right)\right)=0 \text {, and } I_{\lambda_{n}}\left(u\left(\lambda_{n}\right)\right) \rightarrow c_{k} \quad \text { as } n \rightarrow \infty,
$$

where $c_{k} \in\left[d_{k}(2), b_{k}(1)\right]$. In particular, if $\left\{u\left(\lambda_{n}\right)\right\}$ has a convergent subsequence for every $k$, then $I_{1}$ has infinitely many nontrivial critical points $\left\{u_{k}\right\} \in X \backslash\{0\}$ satisfying $I_{1}\left(u_{k}\right) \rightarrow 0^{-}$ as $k \rightarrow \infty$.

Theorem 2.3 Assume that the functional $I_{\lambda}$ defined above satisfies:

$\left(B_{1}\right) I_{\lambda}$ maps bounded sets into bounded sets uniformly for $\lambda \in[1,2]$, and $I_{\lambda}(-u)=I_{\lambda}(u)$ for all $(\lambda, u) \in[1,2] \times X$

$\left(B_{2}\right) B(u) \geq 0, A(u) \rightarrow \infty$ or $B(u) \rightarrow \infty$ as $\|u\| \rightarrow \infty($ or $B(u) \leq 0, B(u) \rightarrow-\infty$ as $\|u\| \rightarrow$ $\infty)$;

$\left(B_{3}\right)$ There exist $\rho_{k}>r_{k}>0$ such that

$$
b_{k}(\lambda)=\inf _{u \in Z_{k},\|u\|=r_{k}} I_{\lambda}(u)>a_{k}(\lambda)=\max _{u \in Y_{k},\|u\|=\rho_{k}} I_{\lambda}(u), \quad \forall \lambda \in[1,2]
$$

Then

$$
b_{k}(\lambda) \leq c_{k}(\lambda):=\inf _{\gamma \in \Gamma_{k}} \max _{u \in B_{k}} I_{\lambda}(\gamma(u)), \quad \forall \lambda \in[1,2]
$$

where $B_{k}=\left\{u \in Y_{k}:\|u\| \leq \rho_{k}\right\}$ and $\Gamma_{k}=\left\{\gamma \in C\left(B_{k}, X\right): \gamma\right.$ is odd, $\left.\left.\gamma\right|_{\partial B_{k}=\mathrm{id}}\right\}(k \geq 2)$. Moreover, for almost every $\lambda \in[1,2]$, there exists a sequence $u_{n}^{k}(\lambda)$ such that

$$
\sup _{n}\left\|u_{n}^{k}(\lambda)\right\|<\infty, \quad I_{\lambda}^{\prime}\left(u_{n}^{k}(\lambda)\right) \rightarrow 0 \quad \text { and } \quad I_{\lambda}\left(u_{n}^{k}(\lambda)\right) \rightarrow c_{k}(\lambda) \quad \text { as } n \rightarrow \infty .
$$

\section{Proof of Theorem 1.1}

In this section, we use Theorem 2.2 to complete the proof.

For the notation in Theorem 2.2, we define the functional $A, B$, and $I_{\lambda}$ on our working space $X$ by

$$
A(u)=\frac{1}{p}\|u\|^{p}-\frac{1}{r} \int_{\mathbb{R}^{N}} h_{2}(x)|u|^{r} d x, \quad B(u)=\frac{1}{q} \int_{\mathbb{R}^{N}} h_{1}(x)|u|^{q} d x,
$$


and

$$
\begin{aligned}
I_{\lambda}(u) & =A(u)-\lambda B(u) \\
& =\frac{1}{p}\|u\|^{p}-\frac{1}{r} \int_{\mathbb{R}^{N}} h_{2}(x)|u|^{r} d x-\frac{\lambda}{q} \int_{\mathbb{R}^{N}} h_{1}(x)|u|^{q} d x, \quad \lambda \in[1,2] .
\end{aligned}
$$

Since $X$ is a separable and reflexive Banach space, then there exist $\left\{e_{i}\right\}_{i=1}^{\infty} \subset X$ and $\left\{e_{i}^{*}\right\}_{i=1}^{\infty} \subset$ $X^{*}$ such that

$$
X=\overline{\operatorname{span}\left\{e_{1}, e_{2}, \ldots\right\}}, \quad X^{*}=\overline{\operatorname{span}\left\{e_{1}^{*}, e_{2}^{*}, \ldots\right\}}
$$

and

$$
\left\langle e_{i}^{*}, e_{j}\right\rangle= \begin{cases}1, & \text { if } i=j \\ 0, & \text { if } i \neq j\end{cases}
$$

Let $X_{i}=\mathbb{R} e_{i}$, and $Y_{k}$ and $Z_{k}$ be defined as (2.10).

In the proof of our results, we need the following limits.

Lemma 3.1 Assume $\left(H_{1}\right),\left(H_{2}\right)$, and $\left(H_{3}\right)\left(\right.$ or $\left.\left(H_{3}^{\prime}\right)\right)$, and let

$$
\alpha_{k}:=\sup _{u \in Z_{k},\|u\|=1}\left(\int_{\mathbb{R}^{N}}\left|h_{1} \| u\right|^{q} d x\right)^{\frac{1}{q}}, \quad \beta_{k}:=\sup _{u \in Z_{k},\|u\|=1}\left(\int_{\mathbb{R}^{N}}\left|h_{2} \| u\right|^{r} d x\right)^{\frac{1}{r}},
$$

then $\alpha_{k}, \beta_{k} \rightarrow 0$ as $k \rightarrow \infty$.

Proof It is clear that $0<\alpha_{k+1} \leq \alpha_{k}$, so $\alpha_{k} \rightarrow \alpha_{0} \geq 0$ as $k \rightarrow \infty$. For every $k \in \mathbb{N}^{+}$, taking $u_{k} \in Z_{k}$ such that $\left\|u_{k}\right\|=1$ and

$$
\left(\int_{\mathbb{R}^{N}}\left|h_{1}\right|\left|u_{k}\right|^{q} d x\right)^{\frac{1}{q}}>\frac{1}{2} \alpha_{k}>0
$$

As $X$ is reflexive, $\left\{u_{k}\right\}$ has a weakly convergent subsequence, without loss of generality, suppose $u_{k} \rightarrow u$ weakly in $X$. Then, for every $i \in \mathbb{N}^{+}$, we have

$$
\left\langle e_{i}^{*}, u\right\rangle=\lim _{k \rightarrow \infty}\left\langle e_{i}^{*}, u_{k}\right\rangle=0
$$

which implies that $u=0$ and $u_{k} \rightarrow 0$ weakly in $X$.

Let $\left(H_{2}\right)$ hold, then for any given small $\varepsilon>0$, we may find $R>0$ big enough such that

$$
\left|h_{1}\right|_{L^{\tau}\left(B_{R}^{c}\right)}<\frac{\varepsilon}{2 S_{q \tau^{\prime}}^{q}}
$$

where $B_{R}=\left\{x \in \mathbb{R}^{N}:|x|<r\right\}, B_{R}^{c}=\mathbb{R}^{N} \backslash B_{R}$, and $S_{q \tau^{\prime}}$ is the embedding constant in (2.6). 
If $\frac{p_{s}^{*}}{p_{s}^{*}-q}<\tau \leq \frac{p}{p-q}$, since the embedding $X \hookrightarrow L^{q \tau^{\prime}}\left(B_{R}\right)$ is compact, then $u_{k} \rightarrow 0$ in $L^{q \tau^{\prime}}\left(B_{R}\right)$ and hence there exists $K_{1}>0$ such that

$$
\left|u_{k}\right|_{L^{q \tau^{\prime}}\left(B_{R}\right)}<\frac{\varepsilon}{2\left|h_{1}\right|_{\tau}}
$$

for $k>K_{1}$.

Using (3.8) and (3.9), for all $k>K_{1}$, we get

$$
\begin{aligned}
\int_{\mathbb{R}^{N}}\left|h_{1}\right|\left|u_{k}\right|^{q} d x & =\int_{B_{R}}\left|h_{1}\right|\left|u_{k}\right|^{q} d x+\int_{B_{R}^{c}}\left|h_{1}\right|\left|u_{k}\right|^{q} d x \\
& \leq\left|h_{1}\right|_{L^{\tau}\left(B_{R}\right)}\left|u_{k}\right|_{L^{q \tau^{\prime}\left(B_{R}\right)}}^{q}+\left|h_{1}\right|_{L^{\tau}\left(B_{R}^{c}\right)}\left|u_{k}\right|_{L^{q \tau^{\prime}\left(B_{R}^{c}\right)}}^{q} \\
& \leq\left|h_{1}\right|_{\tau}\left|u_{k}\right|_{L^{q \tau^{\prime}\left(B_{R}\right)}}^{q}+\left|h_{1}\right|_{L^{\tau}\left(B_{R}^{c}\right)}\left|u_{k}\right|_{q \tau^{\prime}}^{q} \\
& \leq \varepsilon .
\end{aligned}
$$

If $\tau=\frac{p_{s}^{*}}{p_{s}^{*}-q}$, since $u_{k} \rightarrow 0$ in $E$ and the compact embedding $X \hookrightarrow L^{t}\left(B_{R}\right)\left(1 \leq t<p_{s}^{*}\right)$, we can assume that $u_{k} \rightarrow 0$ a.e. in $B_{R}$. For each measurable subset $\Omega \subset B_{R}$, we have

$$
\int_{\Omega}\left|h_{1}\right|\left|u_{k}\right|^{q} d x \leq\left|h_{1}\right|_{L^{\tau}(\Omega)}\left|u_{k}\right|_{L^{p_{S}^{*}(\Omega)}}^{q} \leq C\left|h_{1}\right|_{L^{\tau}(\Omega)} .
$$

Then $\left\{\left|h_{1}\right|\left|u_{k}\right|^{q}\right\}$ is uniformly integrable, and the Vitali convergence theorem implies

$$
\lim _{k \rightarrow \infty} \int_{B_{R}}\left|h_{1}\right|\left|u_{k}\right|^{q} d x=0
$$

So, for $k$ big enough, (3.10) still holds.

Then, from (3.6) and (3.10), we conclude that $\alpha_{k} \rightarrow 0$ as $k \rightarrow \infty$.

Assume $\left(H_{3}\right)$. Since $\mu \in\left[\frac{p_{s}^{*}}{p_{s}^{*}-r}, \infty\right)$ implies $p<r \mu^{\prime} \leq p_{s}^{*}$, arguing as in the above proof, one has $\beta_{k} \rightarrow 0$ as $k \rightarrow \infty$.

Similarly, if assumption $\left(H_{3}^{\prime}\right)$ holds, it follows

$$
\begin{aligned}
\int_{\mathbb{R}^{N}}\left|h_{2}\right|\left|u_{k}\right|^{r} d x & =\int_{B_{R}}\left|h_{2}\right|\left|u_{k}\right|^{r} d x+\int_{B_{R}^{c}}\left|h_{2}\right|\left|u_{k}\right|^{r} d x \\
& \leq\left|h_{2}\right|_{L^{\infty}\left(\mathbb{R}^{N}\right)}\left|u_{k}\right|_{L^{r}\left(B_{R}\right)}^{r}+\left|h_{2}\right|_{L^{\infty}\left(B_{R}^{c}\right)}\left|u_{k}\right|_{L^{r}\left(B_{R}^{c}\right)}^{r}
\end{aligned}
$$

Since $h_{2}(x) \in L^{\infty}\left(\mathbb{R}^{N}\right)$ and $h_{2}(x) \rightarrow 0$ as $|x| \rightarrow \infty, \beta_{k} \rightarrow 0$ can be obtained in a similar way, and we complete the proof.

In order to apply Theorem 2.2, we give the following lemma first.

Lemma 3.2 Let $\left(H_{1}\right),\left(H_{2}\right)$, and $\left(H_{3}\right)\left(\right.$ or $\left.\left(H_{3}^{\prime}\right)\right)$ hold. Then there exist two sequences $0<r_{k}<$ $\rho_{k} \rightarrow 0$ as $k \rightarrow \infty$ such that

$$
a_{k}(\lambda)=\inf _{u \in Z_{k},\|u\|=\rho_{k}} I_{\lambda}(u) \geq 0, \quad b_{k}(\lambda)=\max _{u \in Y_{k},\|u\|=r_{k}} I_{\lambda}(u)<0, \quad \forall \lambda \in[1,2]
$$


and

$$
d_{k}(\lambda)=\inf _{u \in Z_{k},\|u\| \leq \rho_{k}} I_{\lambda}(u) \rightarrow 0, \quad \text { as } k \rightarrow \infty \text { uniformly for } \lambda \in[1,2] .
$$

Proof From Lemma 3.1 we see that, for every $u \in Z_{k}$, it holds

$$
\int_{\mathbb{R}^{N}}\left|h_{1}\right||u|^{q} d x \leq\left(\alpha_{k}\|u\|\right)^{q}, \quad \int_{\mathbb{R}^{N}}\left|h_{2}\right||u|^{r} d x \leq\left(\beta_{k}\|u\|\right)^{r} .
$$

Thus

$$
I_{\lambda}(u) \geq \frac{1}{p}\|u\|^{p}-\frac{1}{r} \beta_{k}^{r}\|u\|^{r}-\frac{\lambda}{q} \alpha_{k}^{q}\|u\|^{q} .
$$

Fix $K_{2}>0$ big enough such that $\frac{1}{r} \beta_{k}^{r}<\frac{1}{2 p}$ for $k>K_{2}$, then for $u \in Z_{k}$ and $\|u\|<1$, we have

$$
I_{\lambda}(u) \geq \frac{1}{p}\|u\|^{p}-\frac{1}{2 p}\|u\|^{r}-\frac{\lambda}{q} \alpha_{k}^{q}\|u\|^{q} \geq \frac{1}{2 p}\|u\|^{p}-\frac{2}{q} \alpha_{k}^{q}\|u\|^{q} .
$$

If we choose $\rho_{k}=\left(8 p \alpha_{k}^{q} / q\right)^{\frac{1}{p-q}}$, then $\rho_{k} \rightarrow 0^{+}$as $k \rightarrow \infty$ and for any $u \in Z_{k}$ with $\|u\|=\rho_{k}$, we get that

$$
I_{\lambda}(u) \geq \frac{1}{4 p}\left(8 p \alpha_{k}^{q} / q\right)^{\frac{p}{p-q}}>0 .
$$

This inequality implies that

$$
a_{k}(\lambda)=\inf _{u \in Z_{k},\|u\|=\rho_{k}} I_{\lambda}(u)>0, \quad \forall \lambda \in[1,2] .
$$

In addition, for all $\lambda \in[1,2], k>K_{2}$ and $u \in Z_{k}$ with $\|u\| \leq \rho_{k}$, we have

$$
I_{\lambda}(u) \geq-\frac{2}{q} \alpha_{k}^{q}\|u\|^{q} \geq-\frac{2}{q} \alpha_{k}^{q} \rho_{k}^{q} \rightarrow 0, \quad \text { as } k \rightarrow \infty .
$$

Obviously,

$$
d_{k}(\lambda)=\inf _{u \in Z_{k},\|u\| \leq \rho_{k}} I_{\lambda}(u) \leq I_{\lambda}(0)=0
$$

So we have $d_{k}(\lambda) \rightarrow 0$ as $k \rightarrow \infty$ uniformly for $\lambda \in[1,2]$.

For all $u \in Y_{k}, \lambda \in[1,2]$, by the equivalence of any norm in a finite dimensional space, we can derive

$$
\begin{aligned}
I_{\lambda}(u) & \leq \frac{1}{p}\|u\|^{p}+\frac{1}{r} \int_{\mathbb{R}^{N}}\left|h_{2}\right||u|^{r} d x-\frac{1}{q} \int_{\mathbb{R}^{N}} h_{1}(x)|u|^{q} d x \\
& \leq \frac{1}{p}\|u\|^{p}+\frac{C_{1}}{r}\|u\|^{r}-\frac{C_{2}}{q}\|u\|^{q},
\end{aligned}
$$

where $C_{1} \geq 0, C_{2}>0$. Notice $q<p<r$, so we can choose $r_{k}>0$ small enough satisfying $r_{k}<\rho_{k}$ such that

$$
b_{k}(\lambda)=\max _{u \in Y_{k},\|u\|=r_{k}} I_{\lambda}(u)<0 .
$$

The proof is completed. 
Proof of Theorem 1.1 At first, we confirm conditions $\left(A_{1}\right)-\left(A_{2}\right)$ in Theorem 2.2. It follows from $\left(H_{1}\right)-\left(H_{3}\right)$ that $I_{\lambda}$ maps bounded sets into bounded sets uniformly for $\lambda \in[1,2]$. Evidently, $I_{\lambda}(-u)=I_{\lambda}(u)$ for all $(\lambda, u) \in[1,2] \times X . B(u) \geq 0$ for all $u \in X$, and by the equivalence of any norm in a finite dimensional space, we know that $B(u) \rightarrow \infty$ as $\|u\| \rightarrow \infty$ on any finite dimensional subspace of $X$. So $\left(A_{1}\right)$ and $\left(A_{2}\right)$ hold.

From Lemma 3.2, we see that $\left(A_{3}\right)$ in Theorem 2.2 are verified. Consequently, for any $k \in \mathbb{Z}^{+}$, there exist $\lambda_{n} \rightarrow 1, u\left(\lambda_{n}\right) \in Y_{n}$ such that

$$
\left.I_{\lambda_{n}}^{\prime}\right|_{Y_{n}}\left(u\left(\lambda_{n}\right)\right)=0, \quad \text { and } \quad I_{\lambda_{n}}\left(u\left(\lambda_{n}\right)\right) \rightarrow c_{k} \in\left[d_{k}(2), b_{k}(1)\right] \quad \text { as } n \rightarrow \infty
$$

Equation (3.23) implies $I_{\lambda_{n}}^{\prime}\left(u\left(\lambda_{n}\right)\right) \rightarrow 0$ as $n \rightarrow \infty$. Then, for large $n$, from $\left(H_{1}\right)-\left(H_{3}\right)$ and (2.6) we have

$$
\begin{aligned}
c+1+\left\|u\left(\lambda_{n}\right)\right\| & \geq I_{\lambda_{n}}\left(u\left(\lambda_{n}\right)\right)-\frac{1}{r} I_{\lambda_{n}}^{\prime}\left(u\left(\lambda_{n}\right)\right) u\left(\lambda_{n}\right) \\
& =\left(\frac{1}{p}-\frac{1}{r}\right)\left\|u\left(\lambda_{n}\right)\right\|^{p}-\lambda_{n}\left(\frac{1}{q}-\frac{1}{r}\right) \int_{\mathbb{R}^{N}} h_{1}(x)\left|u\left(\lambda_{n}\right)\right|^{q} d x \\
& \geq\left(\frac{1}{p}-\frac{1}{r}\right)\left\|u\left(\lambda_{n}\right)\right\|^{p}-2 S_{q \tau^{\prime}}^{q}\left(\frac{1}{q}-\frac{1}{r}\right)\left|h_{1}\right|_{\tau}\left\|u\left(\lambda_{n}\right)\right\|^{q} .
\end{aligned}
$$

Since $p>1, p>q>0$, the above inequality implies that $\left\{u\left(\lambda_{n}\right)\right\}$ is bounded in $X$.

Then there exist a constant $M>0$ and $u \in X$, and a subsequence $\left\{u\left(\lambda_{n}\right)\right\}$, denoted by $\left\{u_{n}\right\}$, such that $\|u\|,\left\|u_{n}\right\| \leq M$ and $u_{n} \rightarrow u$ weakly in $X$. Arguing as in the proof of Lemma 3.1, we have

$$
\lim _{n \rightarrow \infty} \int_{\mathbb{R}^{N}}\left|h_{1}\right|\left|u_{n}-u\right|^{q} d x=0, \quad \lim _{n \rightarrow \infty} \int_{\mathbb{R}^{N}}\left|h_{2}\right|\left|u_{n}-u\right|^{r} d x=0 .
$$

Using the Hölder inequality, we have

$$
\int_{\mathbb{R}^{N}}\left|h_{1}\right|\left|u_{n}\right|^{q-2} u_{n}\left(u_{n}-u\right) d x \leq\left(\int_{\mathbb{R}^{N}}\left|h_{1}\right|\left|u_{n}\right|^{q} d x\right)^{\frac{q-1}{q}}\left(\int_{\mathbb{R}^{N}}\left|h_{1}\right|\left|u_{n}-u\right|^{q} d x\right)^{\frac{1}{q}}
$$

and

$$
\int_{\mathbb{R}^{N}}\left|h_{2}\right|\left|u_{n}\right|^{r-2} u_{n}\left(u_{n}-u\right) d x \leq\left(\int_{\mathbb{R}^{N}}\left|h_{2}\right|\left|u_{n}\right|^{q} d x\right)^{\frac{r-1}{r}}\left(\int_{\mathbb{R}^{N}}\left|h_{2}\right|\left|u_{n}-u\right|^{r} d x\right)^{\frac{1}{r}} .
$$

Then (3.25) implies

$$
\begin{aligned}
& \lim _{n \rightarrow \infty} \int_{\mathbb{R}^{N}} h_{1}(x)\left|u_{n}\right|^{q-2} u_{n}\left(u_{n}-u\right) d x=0, \\
& \lim _{n \rightarrow \infty} \int_{\mathbb{R}^{N}} h_{2}(x)\left|u_{n}\right|^{r-2} u_{n}\left(u_{n}-u\right) d x=0 .
\end{aligned}
$$


Denote

$$
\begin{aligned}
P_{n}:= & I_{\lambda_{n}}^{\prime}\left(u_{n}\right)\left(u_{n}-u\right) \\
= & \int_{\mathbb{R}^{N} \times \mathbb{R}^{N}} \frac{\left|u_{n}(x)-u_{n}(y)\right|^{p-2}\left(u_{n}(x)-u_{n}(y)\right)\left(\left(u_{n}(x)-u(x)\right)-\left(u_{n}(y)-u(y)\right)\right)}{|x-y|^{N+s p}} d x d y \\
& +\int_{\mathbb{R}^{N}} V(x)\left|u_{n}\right|^{p-2} u_{n}\left(u_{n}-u\right) d x+\int_{\mathbb{R}^{N}} h_{1}(x)\left|u_{n}\right|^{q-2} u_{n}\left(u_{n}-u\right) d x \\
& +\int_{\mathbb{R}^{N}} h_{2}(x)\left|u_{n}\right|^{r-2} u_{n}\left(u_{n}-u\right) d x
\end{aligned}
$$

and

$$
\begin{aligned}
Q_{n}:= & \int_{\mathbb{R}^{N} \times \mathbb{R}^{N}} \frac{|u(x)-u(y)|^{p-2}(u(x)-u(y))\left(\left(u_{n}(x)-u(x)\right)-\left(u_{n}(y)-u(y)\right)\right)}{|x-y|^{N+s p}} d x d y \\
& +\int_{\mathbb{R}^{N}} V(x)|u|^{p-2} u\left(u_{n}-u\right) d x .
\end{aligned}
$$

Then the fact $I_{\lambda_{n}}^{\prime}\left(u\left(\lambda_{n}\right)\right) \rightarrow 0$ shows that $P_{n} \rightarrow 0$ as $n \rightarrow \infty$. Moreover, $\left\{u_{n}\right\}$ is a bounded sequence and $u_{n} \rightarrow u$ in $X$, which imply $Q_{n} \rightarrow 0$.

Equations (3.29) and (3.30) show that, for large $n$,

$$
\begin{aligned}
& \int_{\mathbb{R}^{N} \times \mathbb{R}^{N}} \frac{\left|u_{n}(x)-u_{n}(y)\right|^{p-2}\left(u_{n}(x)-u_{n}(y)\right)-|u(x)-u(y)|^{p-2}(u(x)-u(y))}{|x-y|^{N+s p}} \\
& \quad \times\left(\left(u_{n}(x)-u(x)\right)-\left(u_{n}(y)-u(y)\right)\right) d x d y \\
& \quad+\int_{\mathbb{R}^{N}} V(x)\left(\left|u_{n}\right|^{p-2} u_{n}-|u|^{p-2} u\right)\left(u_{n}-u\right) d x \\
& =P_{n}-Q_{n}+o_{n}(1) \rightarrow 0 .
\end{aligned}
$$

By using the standard inequalities (see [23]) given by

$$
\left\langle|\xi|^{p-2} \xi-|\eta|^{p-2} \eta, \xi-\eta\right\rangle \geq C_{p}|\xi-\eta|^{p}, \quad p \geq 2, \forall \xi, \eta \in \mathbb{R}^{N}
$$

and

$$
\left\langle|\xi|^{p-2} \xi-|\eta|^{p-2} \eta, \xi-\eta\right\rangle \geq C_{p}|\xi-\eta|^{2}(|\xi|+|\eta|)^{p-2}, \quad 1<p<2, \forall \xi, \eta \in \mathbb{R}^{N}
$$

where $C_{p}$ is a positive constant and $\langle\cdot, \cdot\rangle$ denotes the inner product in $\mathbb{R}^{N}$, we can easily deduce that $\left\|u_{n}-u\right\| \rightarrow 0$ as $n \rightarrow \infty$. Now, from Theorem 2.2, we see that $I=I_{1}$ possesses infinitely many nontrivial critical points $u_{k}$ for $k \in \mathbb{Z}^{+}$satisfying $I\left(u_{k}\right) \rightarrow 0^{-}$as $k \rightarrow \infty$. Therefore, problem (1.1) possesses infinitely many nontrivial solutions, the proof of Theorem 1.1 is completed.

\section{Proof of Theorem 1.2}

For the notation in Theorem 2.2, we define the functional $A, B$ and $I_{\lambda}$ on our working space $X$ by

$$
A(u)=\frac{1}{p}\|u\|^{p}-\frac{1}{q} \int_{\mathbb{R}^{N}} h_{1}(x)|u|^{q} d x, \quad B(u)=\frac{1}{r} \int_{\mathbb{R}^{N}} h_{2}(x)|u|^{r} d x,
$$


and

$$
\begin{aligned}
I_{\lambda}(u) & =A(u)-\lambda B(u) \\
& =\frac{1}{p}\|u\|^{p}-\frac{1}{q} \int_{\mathbb{R}^{N}} h_{1}(x)|u|^{q} d x-\frac{\lambda}{r} \int_{\mathbb{R}^{N}} h_{2}(x)|u|^{r} d x, \quad \lambda \in[1,2] .
\end{aligned}
$$

Lemma 4.1 Let $\left(H_{1}\right)-\left(H_{3}\right)$ hold and $h_{2}(x)>0$. Then there exist two sequences $\rho_{k}>r_{k}>0$ such that

$$
b_{k}(\lambda)=\inf _{u \in Z_{k},\|u\|=r_{k}} I_{\lambda}(u)>a_{k}(\lambda)=\max _{u \in Y_{k},\|u\|=\rho_{k}} I_{\lambda}(u), \quad \forall \lambda \in[1,2] .
$$

Proof Similar to the beginning of the proof of Lemma 3.2, by (3.14) we have

$$
I_{\lambda}(u) \geq \frac{1}{p}\|u\|^{p}-\frac{1}{q} \alpha_{k}^{q}\|u\|^{q}-\frac{2}{r} \beta_{k}^{r}\|u\|^{r} .
$$

Fix $K_{3}>0$ big enough such that $\frac{2}{q} \alpha_{k}^{q}<\frac{1}{2 p}$ for $k>K_{3}$, then for $u \in Z_{k}$ and $\|u\|>1$, we have

$$
I_{\lambda}(u) \geq \frac{1}{2 p}\|u\|^{p}-\frac{2}{r} \beta_{k}^{r}\|u\|^{r} .
$$

If we choose $r_{k}=\left(\frac{r}{8 p \beta_{k}^{r}}\right)^{\frac{1}{r-p}}$, then $r_{k} \rightarrow \infty$ as $k \rightarrow \infty$, and for any $u \in Z_{k}$ with $\|u\|=r_{k}$, we get that

$$
I_{\lambda}(u) \geq \frac{1}{4 p} r_{k}^{p}>0
$$

This inequality implies that

$$
b_{k}(\lambda)=\inf _{u \in Z_{k},\|u\|=r_{k}} I_{\lambda}(u) \geq \frac{1}{4 p} r_{k}^{p}>0, \quad \forall \lambda \in[1,2] .
$$

For all $u \in Y_{k}, \lambda \in[1,2]$, by the equivalence of any norm in a finite dimensional space, we can derive

$$
I_{\lambda}(u) \leq \frac{1}{p}\|u\|^{p}+C_{1}\|u\|^{q}-C_{2}\|u\|^{r}
$$

where $C_{1} \geq 0, C_{2}>0$. Notice $q<p<r$, then $I_{\lambda}(u) \rightarrow-\infty$ as $\|u\| \rightarrow \infty$. So we can choose $\rho_{k}>r_{k}$ big enough such that

$$
a_{k}(\lambda)=\max _{u \in Y_{k},\|u\|=\rho_{k}} I_{\lambda}(u)<0
$$

The proof is completed.

Proof of Theorem 1.2 We complete the proof by Theorem 2.3. Let us verify the conditions in Theorem 2.3 firstly.

$\left(H_{1}\right)-\left(H_{3}\right)$ imply that $I_{\lambda}$ maps bounded sets into bounded sets uniformly for $\lambda \in[1,2]$. Moreover, $I_{\lambda}(-u)=I_{\lambda}(u)$ for all $u \in X$ and $\lambda \in[1,2]$. So condition $\left(B_{1}\right)$ of Theorem 2.3 holds. 
Evidently, $B(u) \geq 0$ for all $u \in X$, and $A(u) \rightarrow \infty$ as $\|u\| \rightarrow \infty$. $\left(B_{2}\right)$ in Theorem 2.3 is verified.

From Lemma 4.1, we see that condition $\left(B_{3}\right)$ has been verified. Therefore, by Theorem 2.3, for a.e. $\lambda \in[1,2]$, there exists a sequence $u_{n}^{k}(\lambda)_{n=1}^{\infty}$ such that

$$
\sup _{n}\left\|u_{n}^{k}(\lambda)\right\|<\infty, \quad I_{\lambda}^{\prime}\left(u_{n}^{k}(\lambda)\right) \rightarrow 0, \quad \text { and } \quad I_{\lambda}\left(u_{n}^{k}(\lambda)\right) \rightarrow c_{k}(\lambda) \quad \text { as } n \rightarrow \infty .
$$

Moreover, by Theorem 2.3 and (4.6) we see that

$$
c_{k}(\lambda) \geq b_{k}(\lambda) \geq \frac{1}{4 p}\left(\frac{r}{8 p \beta_{k}^{r}}\right)^{\frac{p}{r-p}}:=\bar{b}_{k} \rightarrow \infty \quad \text { as } k \rightarrow \infty .
$$

Since

$$
c_{k}(\lambda)=\inf _{\gamma \in \Gamma_{k}} \max _{u \in B_{k}} I_{\lambda}(\gamma(u)) \leq \max _{u \in B_{k}} I_{1}(u):=\bar{c}_{k} .
$$

Then we have

$$
\bar{b}_{k} \leq c_{k} \leq \bar{c}_{k}
$$

If we choose a sequence $\lambda_{m} \rightarrow 1$, then (4.9) implies that the sequence $\left\{u_{n}^{k}\left(\lambda_{m}\right)\right\}_{n=1}^{\infty}$ is bounded. Using similar arguments as those in the proof of Theorem 1.1, we can prove that the sequence $\left\{u_{n}^{k}\left(\lambda_{m}\right)\right\}_{n=1}^{\infty}$ has a strong convergent subsequence as $n \rightarrow \infty$. Thus we may assume that $u_{n}^{k}\left(\lambda_{m}\right) \rightarrow u^{k}\left(\lambda_{m}\right)$ in $X$ as $n \rightarrow \infty$ for every $m \in \mathbb{N}$. Moreover, by (4.9) and (4.12) we have

$$
I_{\lambda}^{\prime}\left(u^{k}\left(\lambda_{m}\right)\right)=0 \quad \text { and } \quad I_{\lambda_{m}}\left(u^{k}\left(\lambda_{m}\right)\right) \in\left[\bar{b}_{k}, \bar{c}_{k}\right]
$$

As in the proof of Theorem 1.1, we can get the boundedness of $\left\{u^{k}\left(\lambda_{m}\right)\right\}_{m=1}^{\infty}$ and prove it possesses a strong convergent subsequence with the limit $u^{k} \in X$. Therefore, the limit $u^{k} \in X$ is a critical point of $I$ with $I\left(u^{k}\right) \in\left[\bar{b}_{k}, \bar{c}_{k}\right]$. Since $\bar{b}_{k} \rightarrow \infty$ as $k \rightarrow \infty$, we get infinitely many nontrivial critical points of $I$. Consequently, problem (1.1) possesses infinitely many nontrivial solutions with high energy. The proof is completed.

\section{Acknowledgements}

The authors would like to express their sincere gratitude to the anonymous reviewer for their valuable comments and suggestions which improved the presentation of this manuscript.

Funding

Project supported by NSFC (No. 11801492) and NSFJS Grant (BK 20170472).

Abbreviations

Not applicable.

Availability of data and materials

Not applicable.

Competing interests

The authors declare that they have no competing interests. 


\section{Author details}

'School of Mathematics and Physics, Yancheng Institute of Technology, Yancheng, P.R. China. ${ }^{2}$ College of Science, Hohai University, Nanjing, P.R. China.

\section{Publisher's Note}

Springer Nature remains neutral with regard to jurisdictional claims in published maps and institutional affiliations.

Received: 20 July 2019 Accepted: 4 March 2020 Published online: 20 March 2020

\section{References}

1. Di Nezza, E., Palatucci, G., Valdinoci, E.: Hitchhiker's guide to the fractional Sobolev spaces. Bull. Sci. Math. 136, 521-573 (2012)

2. Franzina, G., Palatucci, G.: Fractional p-eigenvalues. Riv. Mat. Univ. Parma 5, 315-328 (2014)

3. Xiang, M., Zhang, B., Rădulescu, V.D.: Existence of solutions for perturbed fractional $p$-Laplacian equations. J. Differ. Equ. 260, 1392-1413 (2016)

4. Autuori, G., Pucci, P.: Elliptic problems involving the fractional Laplacian in $\mathbb{R}^{N}$. J. Differ. Equ. 255, 2340-2362 (2013)

5. Khoutir, S., Chen, H.: Existence of infinitely many high energy solutions for a fractional Schrödinger equation in $\mathbb{R}^{N}$. Appl. Math. Lett. 61, 156-162 (2016)

6. Teng, K.M.: Multiple solutions for a class of fractional Schrödinger equations in $\mathbb{R}^{N}$. Nonlinear Anal., Real World Appl. 21, 76-86 (2015)

7. Ge, B.: Multiple solutions of nonlinear Schrödinger equation with the fractional Laplacian. Nonlinear Anal., Real World Appl. 30, 236-247 (2016)

8. Gou, T., Sun, H.: Solutions of nonlinear Schrödinger equation with fractional Laplacian without the Ambrosetti-Rabinowitz condition. Appl. Math. Comput. 257, 409-416 (2015)

9. Perera, K., Squassina, M., Yang, Y.: Critical fractional $p$-Laplacian problems with possibly vanishing potentials. J. Math Anal. Appl. 433(2), 818-831 (2015)

10. Secchi, S.: Ground state solutions for nonlinear fractional Schrödinger equations in $\mathbb{R}^{N}$. J. Math. Phys. 54, 031501 (2013)

11. Chang, X.J., Wang, Z.Q.: Ground state of scalar field equations involving a fractional Laplacian with general nonlinearity. Nonlinearity 26, 479-494 (2013)

12. Felmer, P., Quaas, A., Tan, J.: Positive solutions of the nonlinear Schrödinger equation with the fractional Laplacian. Proc. R. Soc. Edinb., Sect. A 142, 1237-1262 (2012)

13. Secchi, S.: Perturbation results for some nonlinear equations involving fractional operators. Differ. Equ. Appl. 5 221-236 (2013)

14. Secchi, S., Squassina, M.: Soliton dynamics for fractional Schrödinger equations. Appl. Anal. 93, 1702-1729 (2014)

15. Xiang, M., Rădulescu, V.D., Zhang, B.: Fractional Kirchhoff problems with critical Trudinger-Moser nonlinearity. Calc. Var. Partial Differ. Equ. 58(2), Art. 57 (2019)

16. Xiang, M., Rǎdulescu, V.D., Zhang, B.: A critical fractional Choquard-Kirchhoff problem with magnetic field. Commun. Contemp. Math. 21(4), 185004 (2019)

17. Xiang, M., Rǎdulescu, V.D., Zhang, B.: Combined effects for fractional Schrödinger-Kirchhoff systems with critical nonlinearities. ESAIM Control Optim. Calc. Var. 24, 1249-1273 (2018)

18. Xiang, M., Rǎdulescu, V.D., Zhang, B.: Nonlocal Kirchhoff diffusion problems: local existence and blow-up of solutions. Nonlinearity 31, 3228-3250 (2018)

19. Xiang, M., Zhang, B., Rădulescu, V.D.: Superlinear Schrödinger-Kirchhoff type problems involving the fractional p-Laplacian and critical exponent. Adv. Nonlinear Anal. 9, 690-709 (2020)

20. Goyal, S., Sreenadh, K.: Nehari manifold for non-local elliptic operator with concave-convex nonlinearities and sign-changing weight functions. Proc. Indian Acad. Sci. Math. Sci. 125(4), 545-558 (2015)

21. Xiang, M., Zhang, B., Ferrara, M.: Multiplicity results for the non-homogeneous fractional $p$-Kirchhoff equations with concave-convex nonlinearities. Proc. R. Soc. A 471, 20150034 (2015). https://doi.org/10.1098/rspa.2015.0034

22. Zou, W.: Variant fountain theorems and their applications. Manuscr. Math. 104, 343-358 (2001)

23. Dinca, G., Jebelean, P.: Some existence results for a class of nonlinear equations involving a duality mapping. Nonlinear Anal. 46, 347-363 (2001)

\section{Submit your manuscript to a SpringerOpen ${ }^{\circ}$ journal and benefit from:}

- Convenient online submission

- Rigorous peer review

- Open access: articles freely available online

- High visibility within the field

Retaining the copyright to your article

Submit your next manuscript at $>$ springeropen.com 